\title{
Unravelling Melatonin's Varied Antioxidizing Protection of Membrane Lipids Determined by its Spatial Distribution
}

Dongmei Zhang, Chu Gong, Jie Wang, Dong Xing, Lingling Zhao, Danyang Li,

Xinxing Zhang*

College of Chemistry, Key Laboratory of Advanced Energy Materials Chemistry

(Ministry of Education), Renewable Energy Conversion and Storage Center

(ReCAST), Frontiers Science Center for New Organic Matter, Nankai University,

Tianjin, 300071, China.

Corresponding Author

*e-mail: zhangxx@nankai.edu.cn 


\section{Experimental Procedures}

Chemicals and Materials. POPC was purchased from Avanti Polar Lipids (Alabaster, AL, USA) and used without further purification. Melatonin was purchased from SigmaAldrich (powder, $\geq 98 \%$ ) and used without further purification. Ammonium iron ( II) sulfate hexahydrate $\left(\left(\mathrm{NH}_{4}\right)_{2} \mathrm{Fe}\left(\mathrm{SO}_{4}\right)_{2} \cdot 6 \mathrm{H}_{2} \mathrm{O}\right) \quad(99 \%)$ was purchased from Energy Chemical (Shanghai, China) and $\mathrm{H}_{2} \mathrm{O}_{2}(30 \%)$ was obtained from Damao Chemical Reagent (Tianjin, China). HPLC grade methanol was purchased from Fuchen Chemistry Reagent (Tianjin, China). Milli-Q water $\left(18.2 \mathrm{M} \Omega \cdot \mathrm{cm}^{-1}\right)$ used for all experiments was obtained from a Barnstead Easypure II system (Thermo Fisher Scientific, Waltham, MA, USA). $2 \mathrm{mM}$ stock solutions of POPC, melatonin and DIBODIPY are firstly obtained by dissolving the chemicals in methanol and stored at -20 ${ }^{\circ} \mathrm{C}$, respectively. Fenton solutions containing $\left(\mathrm{NH}_{4}\right)_{2} \mathrm{Fe}\left(\mathrm{SO}_{4}\right)_{2} \bullet 6 \mathrm{H}_{2} \mathrm{O}$ and $\mathrm{H}_{2} \mathrm{O}_{2}$ were prepared in water and used freshly.

Online FIDI-MS. The FIDI-MS instrument has been described in detail elsewhere ${ }^{1-6}$. Briefly, a hanging droplet of $\sim 2 \mathrm{~mm}$ o.d. ( $\sim 4 \mu \mathrm{L}$ in volume) is suspended at the end of a stainless-steel capillary between two parallel plate electrodes separated by $6.3 \mathrm{~mm}$. Droplets are formed from liquid fed through the capillary using a motorized syringe pump. The parallel plates are mounted on a translation stage to allow alignment of an aperture in the electrically grounded plate with the atmospheric pressure inlet of an LTQ-XL mass spectrometer (Thermo-Fisher, Waltham, MA). The capillary is mounted on a separate translation stage to place the droplet exactly midway between the two plates and to align with the inlet of the LTQ-XL. Mass spectrometric sampling of the hanging droplet is accomplished by applying a pulsed high voltage (typically 3 to $6 \mathrm{kV}$, $15 \mathrm{~ms}$ duration) to the back parallel plate and to the capillary at half the magnitude applied to the back plate to maintain field homogeneity between the front and back plate. When a sufficiently high voltage is applied, the electrical forces overcome the surface tension of the droplet, resulting in the bipolar ejection of highly charged progeny droplets less than $1 \mu \mathrm{m}$ in diameter from the opposite ends of the suspended droplet. Charged droplets of a specific polarity enter the transfer capillary of the mass spectrometer, resulting in the detection of gas-phase ions.

The hanging droplet in this study is a mixed water solution of POPC $(100 \mu \mathrm{M})$, $\left(\mathrm{NH}_{4}\right)_{2} \mathrm{Fe}\left(\mathrm{SO}_{4}\right)_{2} \cdot 6 \mathrm{H}_{2} \mathrm{O}(100 \mu \mathrm{M}), \mathrm{H}_{2} \mathrm{O}_{2}(1000 \mu \mathrm{M})$ and different concentrations of melatonin or a mixed water solution of POPC $(100 \mu \mathrm{M})$, DI-BODIPY $(5 \mu \mathrm{M})$ and different concentrations of melatonin. After each droplet is formed, we allow $60 \mathrm{~s}$ for the lipid molecules to diffuse to the air-water interface before exposing the droplet to a UV point light $\left(365 \mathrm{~nm}, 1.43 \times 10^{-4} \mathrm{~W} \text {, beam size } 1.5 \mathrm{~mm}, 2.65 \times 10^{14} \text { photon } / \mathrm{s}\right)^{5}$ for the Fenton reactions or a green laser pointer $\left(532 \mathrm{~nm}, 1.68 \times 10^{-5} \mathrm{~W}\right.$, Figure S6, beam size $1.5 \mathrm{~mm}, 4.49 \times 10^{13}$ photon $/ \mathrm{s}$ ) for the photosensitized reactions for a variable reaction time. Individual droplets are then sampled by application of a pulsed high voltage after $0-120 \mathrm{~s}$ of radiation. A positive high voltage is applied to the back plate 
and capillary to detect the POPC, melatonin and relative oxidation products.

Synthesis of DI-BODIPY. The synthetic route of DI-BODIPY is presented in Figure $\mathrm{S} 4$. The compound is synthesized according to a procedure previously reported in literatures ${ }^{7,8}$. Briefly, 4-(chloromethyl) benzoyl chloride $(2.27 \mathrm{~mL}, 19.5 \mathrm{mM})$ and 2, 4dimethylpyrrole $(4 \mathrm{~mL}, 38.9 \mathrm{mM})$ are dissolved in dichloromethane $(150 \mathrm{~mL})$. After stirring at room temperature under nitrogen for $3 \mathrm{~h}$, trimethylamine $(80 \mathrm{~mL})$ is added to the solution. After $1 \mathrm{~h}$, boron trifluoride ethyl ether $(80 \mathrm{~mL})$ is added and stirring continues for another $6 \mathrm{~h}$. The resultant product is purified via silica gel column chromatography using petroleum ether ethyl acetate $(\mathrm{v} / \mathrm{v}, 6: 1)$ as eluent to generate a red solid.

To introduce iodine atom into the BODIPY molecule, excessive amounts $\mathrm{N}$ iodosuccinimide is used to prepare DI-BODIPY. The reaction proceeds in anhydrous $\mathrm{N}, \mathrm{N}$-Dimethylformamide for $24 \mathrm{~h}$ in dark. The final product shows a deep red solid, and the resultant solution is dialyzed against water (MWCO 3500) for 3 days before lyophilization to get DI-BODIPY.

The UV-Vis absorption spectrum of DI-BODIPY is provided in Figure S5. 

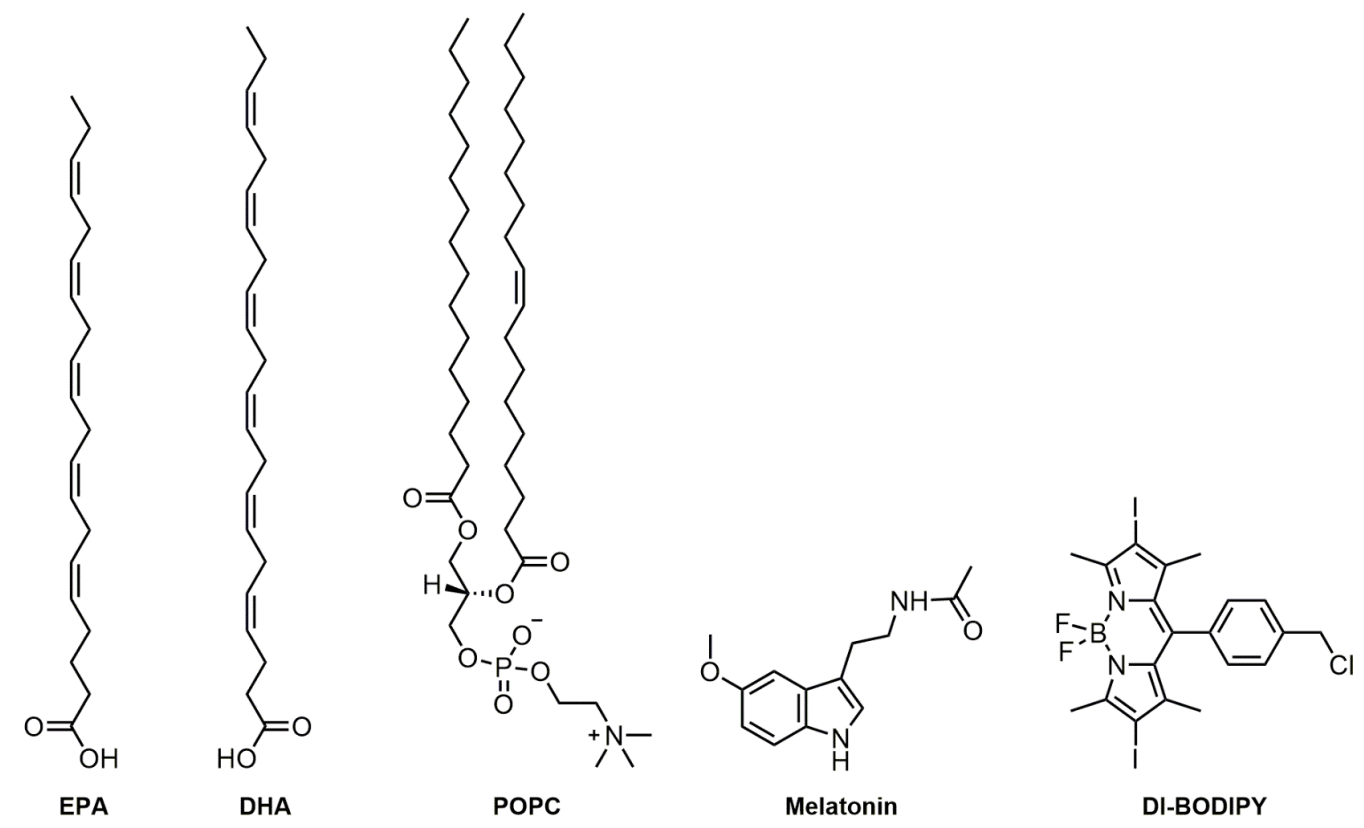

Figure S1. The structures of all the key molecules mentioned in the main text. 


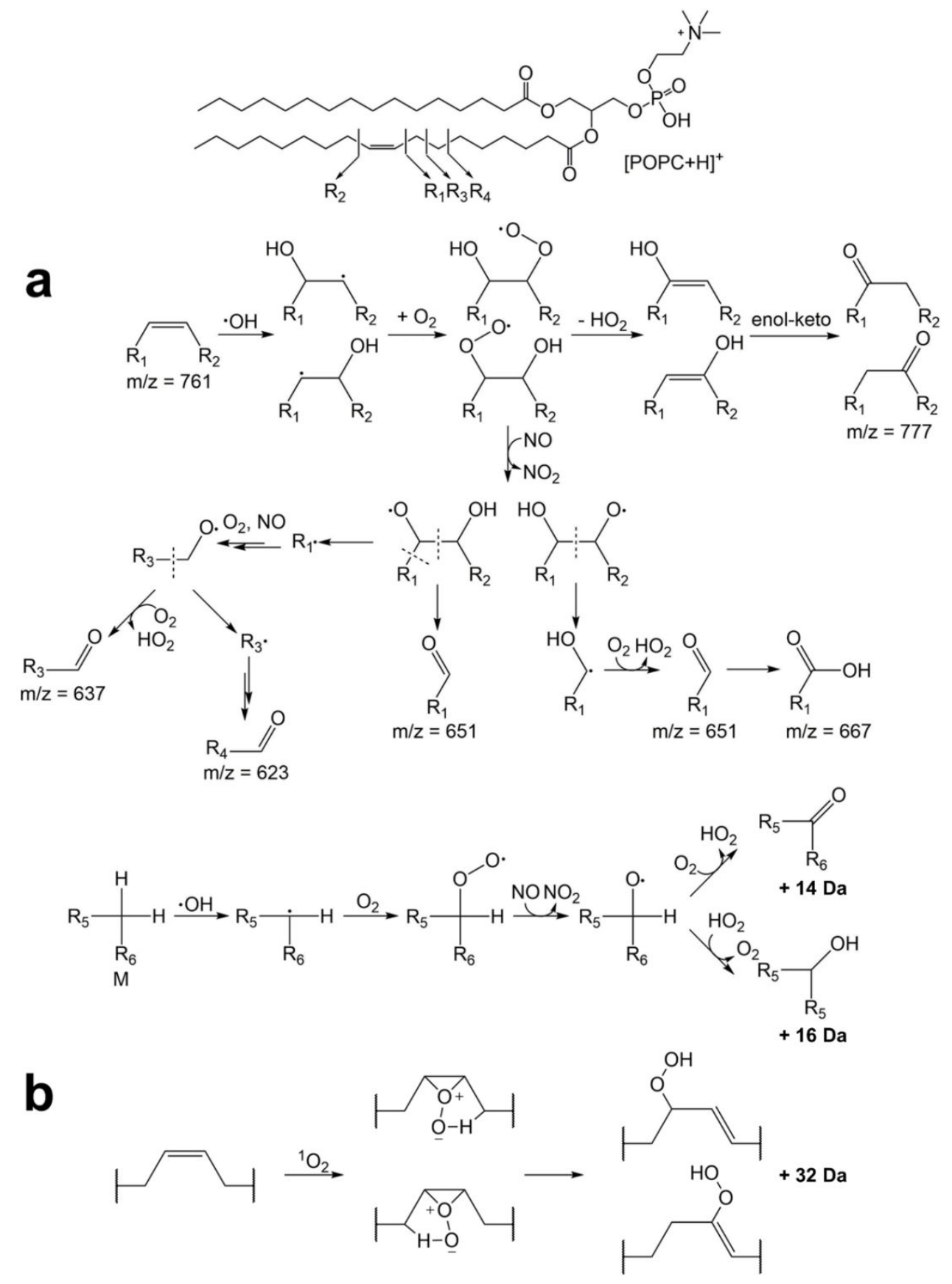

Figure S2. (a) Proposed oxidation mechanisms of the unsaturated and saturated carbons of POPC by $\bullet \mathrm{OH}$ as reported in Reference 5. (b) Proposed peroxidation mechanism of POPC by SO (Reference 6). 

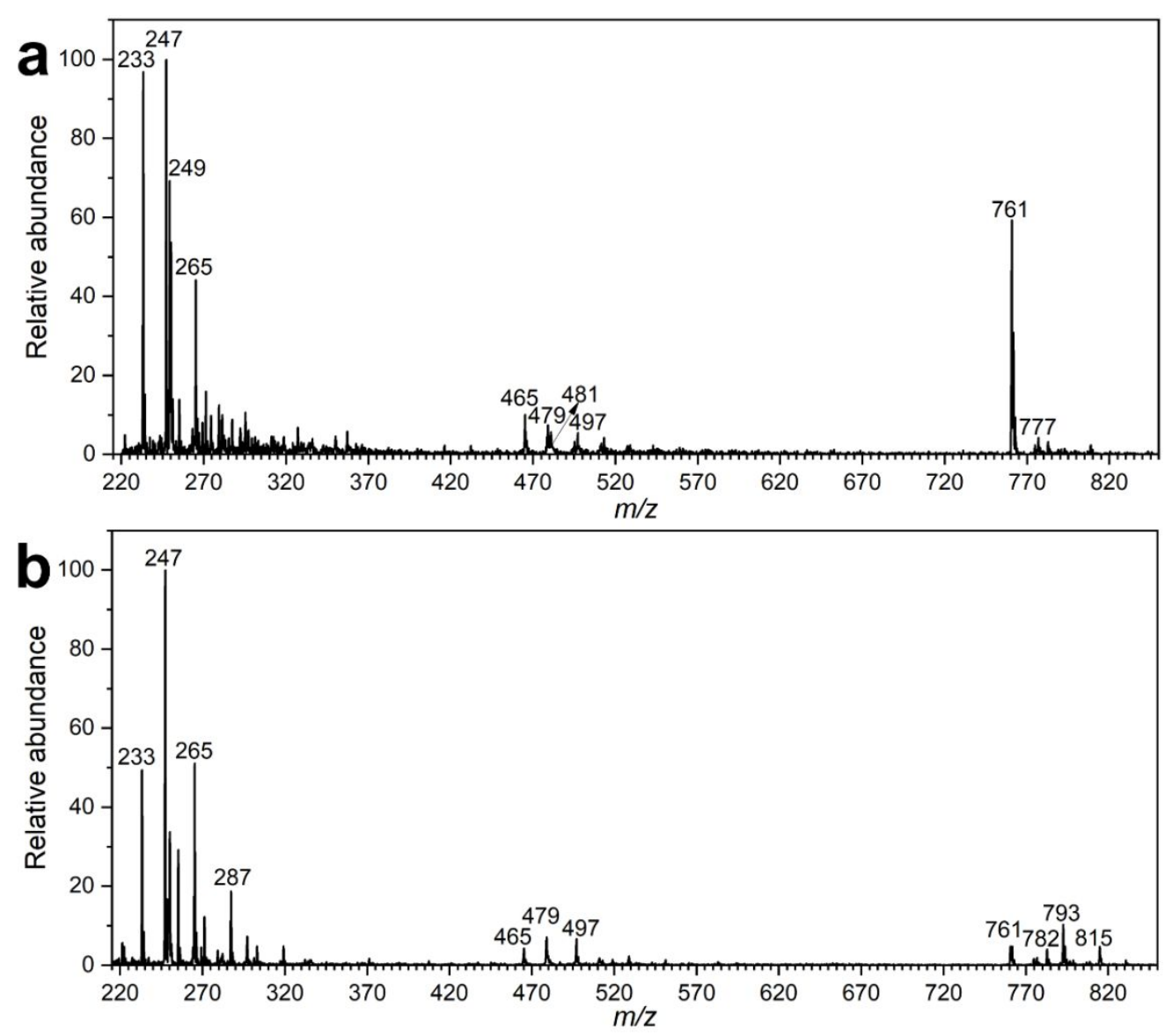

Figure S3. (a) A typical total ion chromatography (TIC) spectrum with $30 \mu \mathrm{M} M, 100$ $\mu \mathrm{M}$ POPC and their $\bullet \mathrm{OH}$ oxidation products after 1 min of the Fenton reaction. (b) A typical TIC spectrum with $30 \mu \mathrm{M}$ M, $100 \mu \mathrm{M}$ POPC and their SO oxidation products after illuminated by $532 \mathrm{~nm}$ for $2 \mathrm{~min}$. 

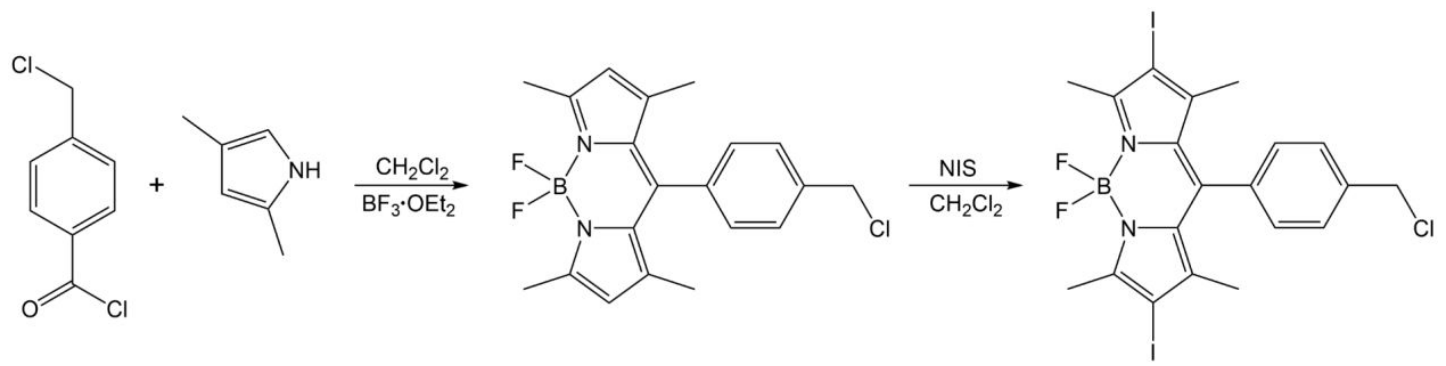

Figure S4. The synthetic route of DI-BODIPY. 


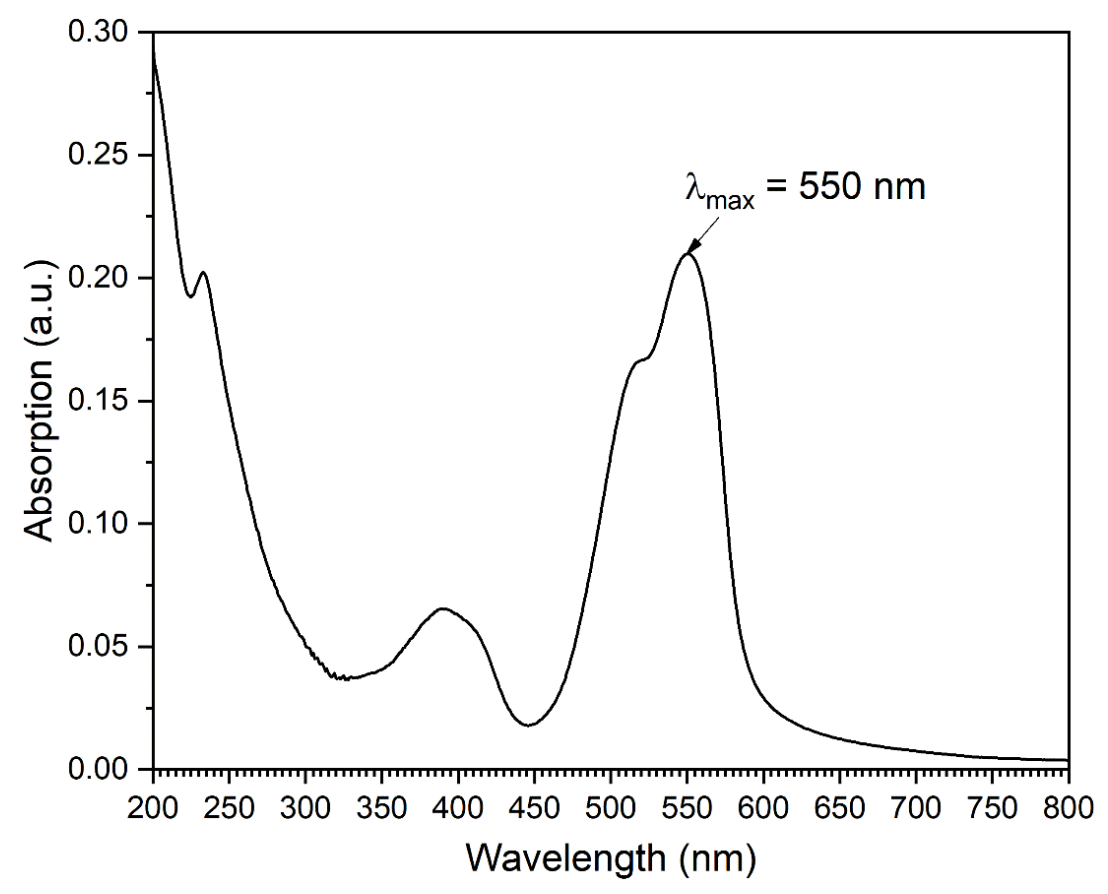

Figure S5. The UV-Vis absorption spectrum of DI-BODIPY. 


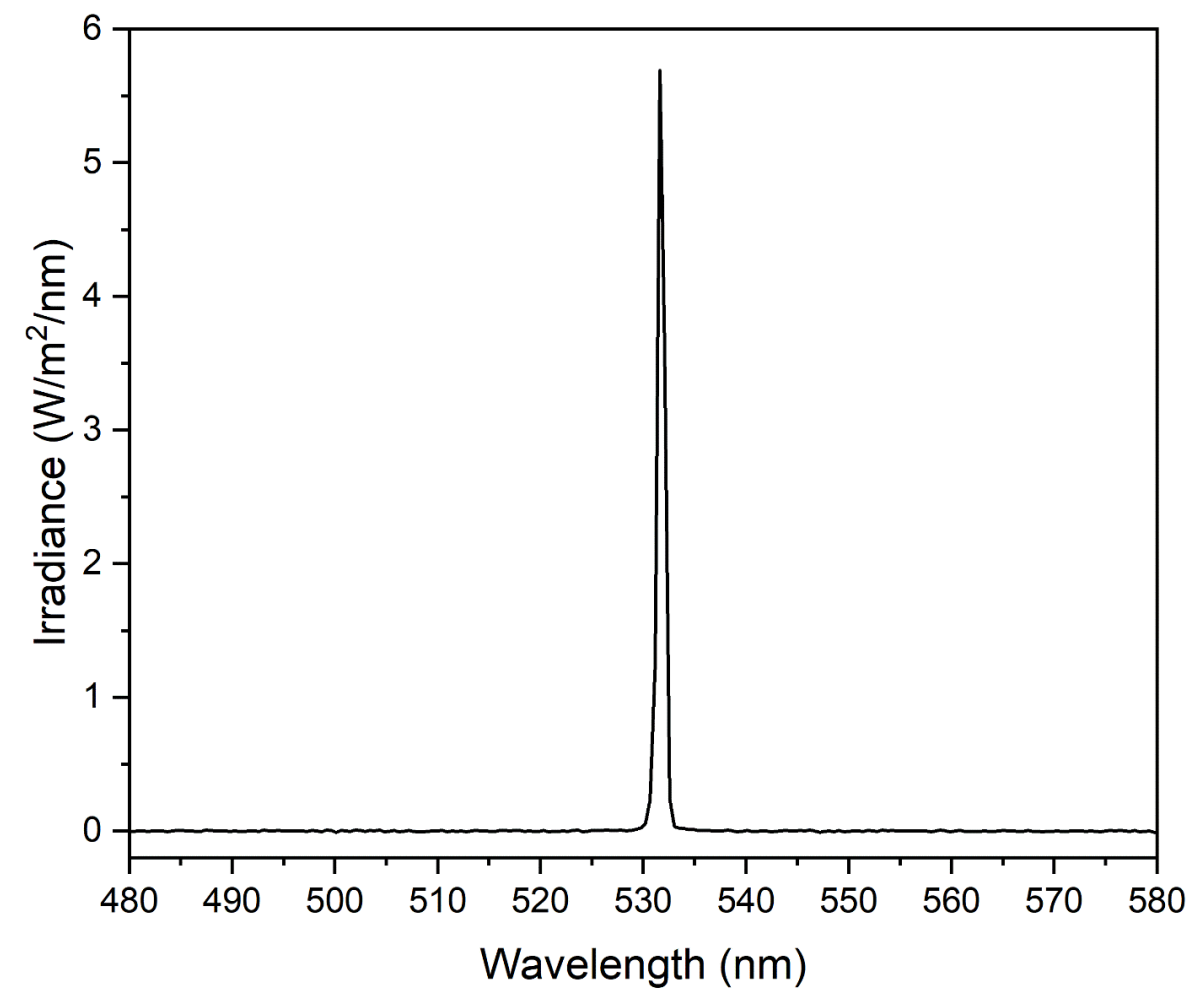

Figure S6. The output of the green laser pointer $(532 \mathrm{~nm})$ used in the current experiment measured by an FX2000-EX UV-VIS spectrometer (Ideaoptics, Shanghai). The energy integrated from 530 to $534 \mathrm{~nm}$ is $5.29 \mathrm{~W} / \mathrm{m}^{2}$, making the energy delivered to a $2 \mathrm{~mm}$ diameter droplet $1.68 \times 10^{-5} \mathrm{~W}$. 

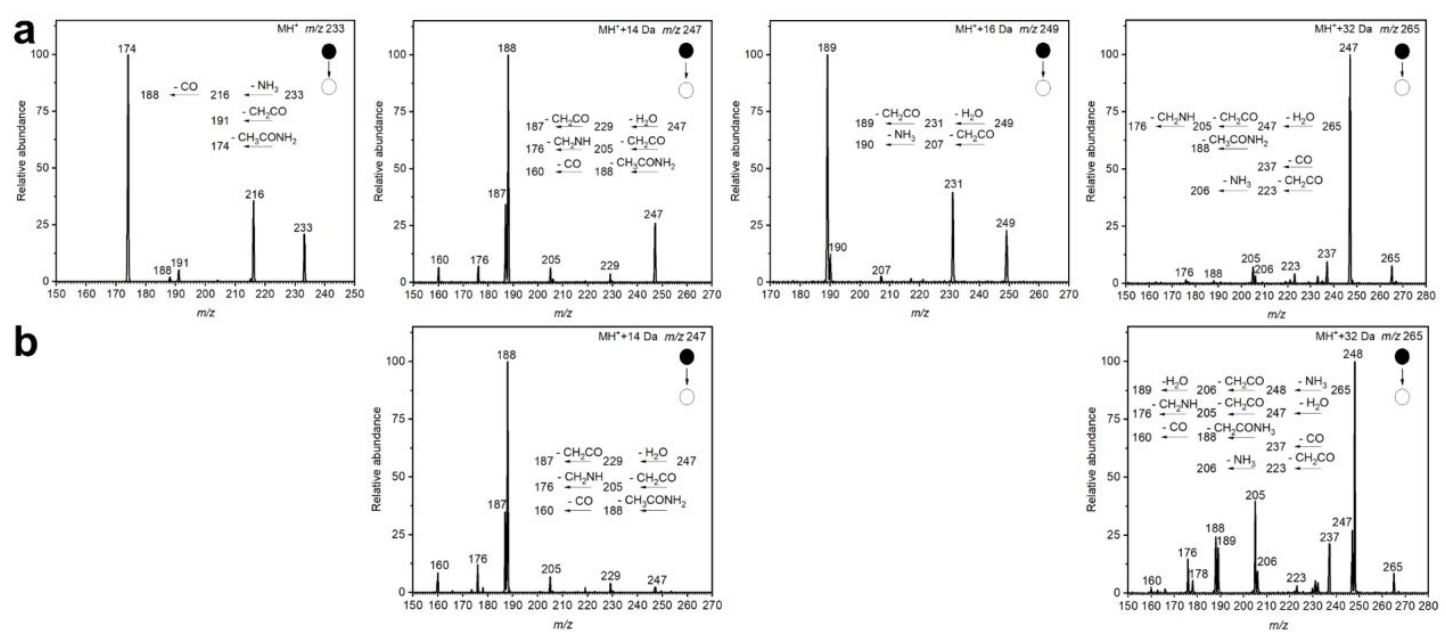

Figure S7. (a) The collision-induced dissociation (CID) spectra of the oxidation products of $\mathrm{M}$ by $\cdot \mathrm{OH}$. (b) The CID spectra of the oxidation products of $\mathrm{M}$ by SO. 


\section{References}

(1) Zhang, X.; Barraza, K. M.; Beauchamp, J. L. Cholesterol Provides

Nonsacrificial Protection of Membrane Lipids from Chemical Damage at Air-Water Interface. Proc. Nat1. Acad. Sci. U. S. A. 2018, 115, 3255-3260.

(2) Zhang, X.; Barraza, K. M.; Upton, K. T.; Beauchamp, J. L. Subtle Changes in Lipid Environment Have Profound Effects on Membrane Oxidation Chemistry. $J$. Am. Chem. Soc. 2018, 140, 17492-17498.

(3) Mu, C.; Wang, J.; Barraza, K. M.; Zhang, X.; Beauchamp, J. L. Mass

Spectrometric Study of Acoustically Levitated Droplets Illuminates Molecular-Level Mechanism of Photodynamic Therapy for Cancer Involving Lipid Oxidation. Angew. Chem. Int. Ed. 2019, 58, 8082-8086.

(4) Geng, W.; Zhang, D.; Gong, C.; Li, Z.; Barraza, K. M.; Beauchamp, J. L.; Guo, D.; Zhang, X. Host-Guest Complexation of Amphiphilic Molecules at the Air-Water Interface Prevents Oxidation by Hydroxyl Radicals and Singlet Oxygen. Angew. Chem. Int. Ed. 2020, 59, 12684-12688.

(5) Zhang, D.; Gong, C.; Wang, J.; Mu, C.; Wang, W.; Zhang, X. Beyond Lipid Peroxidation: Distinct Mechanisms Observed for POPC and POPG Oxidation Initiated by UV-Enhanced Fenton Reactions at the Air-Water Interface. J. Mass Spectrom. 2021, 56, 1-7. 
(5) Mu, C.; Wang, W.; Wang, J.; Gong, C.; Zhang, D.; Zhang, X. Probe-Free Direct Identification of Type I and Type II Photosensitized Oxidation Using Field-Induced Droplet Ionization Mass Spectrometry. Angew. Chem. Int. Ed. 2020, 59, 2151521519.

(7) Nagai, A.; Yoshii, R.; Otsuka, T.; Kokado, K.; Chujo, Y. BODIPY-Based Chain Transfer Agent: Reversibly Thermoswitchable Luminescent Gold Nanoparticle Stabilized by BODIPY-Terminated Water-Soluble Polymer. Langmuir 2010, 26, 15644-15649.

(8) Popere, B. C.; Della Pelle, A. M.; Thayumanavan, S. BODIPY-Based DonorAcceptor П-Conjugated Alternating Copolymers. Macromolecules 2011, 44, 47674776. 